\title{
Online Process Monitoring with Near-Zero Misdetection for Ultrasonic Welding of Lithium-ion Batteries: An Integration of Univariate and Multivariate Methods
}

Weihong Guo $^{1 *}$, Chenhui Shao ${ }^{2}$, Tae Hyung Kim² ${ }^{2}$, S. Jack Hu${ }^{2}$, Jionghua (Judy) Jin ${ }^{1}$, J. Patrick Spicer $^{3}$, and Hui Wang ${ }^{3}$

${ }^{1}$ Department of Industrial and Operations Engineering, University of Michigan, Ann Arbor, MI 48109

${ }^{2}$ Department of Mechanical Engineering, University of Michigan, Ann Arbor, MI 48109

${ }^{3}$ General Motors Company, Global Research \& Development, 30500 Mound Road, Warren, MI 48090

*Corresponding author: E-mail address: graceguo@umich.edu, Tel.: (+1)734-730-5978.

\begin{abstract}
Ultrasonic metal welding is used for joining lithium-ion batteries of electric vehicles. The monitoring of battery joining processes requires near-zero misdetection in order to prevent any battery joints with a low quality connection going into the downstream assembly. The conventional control chart techniques widely used in many process monitoring systems were designed based on a pre-specified false alarm rate. To ensure weld quality and reduce manual inspection at the same time, a near-zero misdetection rate is desired foremost while achieving a
\end{abstract}


low false alarm rate. A monitoring algorithm targeting near-zero misdetection is developed in this article by integrating univariate control charts and the Mahalanobis distance approach. The proposed algorithm is capable of monitoring non-normal multivariate observations with flexible control limits to achieve a near-zero misdetection rate while keeping a low false alarm rate. By implementing this algorithm on the ultrasonic welding process of battery manufacturing, the developed algorithm proves to be effective in achieving near-zero misdetection in process monitoring to ensure battery weld quality. The developed algorithm also shows great potential for monitoring other processes that target at near-zero misdetection.

Keywords: Shewhart control chart, Mahalanobis distance, process monitoring, near-zero misdetection, ultrasonic metal welding, lithium-ion battery 


\section{Introduction}

In recent years, increasing concerns over the environmental impact of the petroleum-based transportation infrastructure and soaring gas price have led to great interest in electric vehicles. Electric vehicles require high-power and high-capacity rechargeable batteries. In manufacturing such batteries, significant challenges exist in creating reliable interconnections between battery cells, between modules, and between modules and control units. Such connections must possess reliable electrical conductivity and sufficient mechanical strength to ensure battery performance.

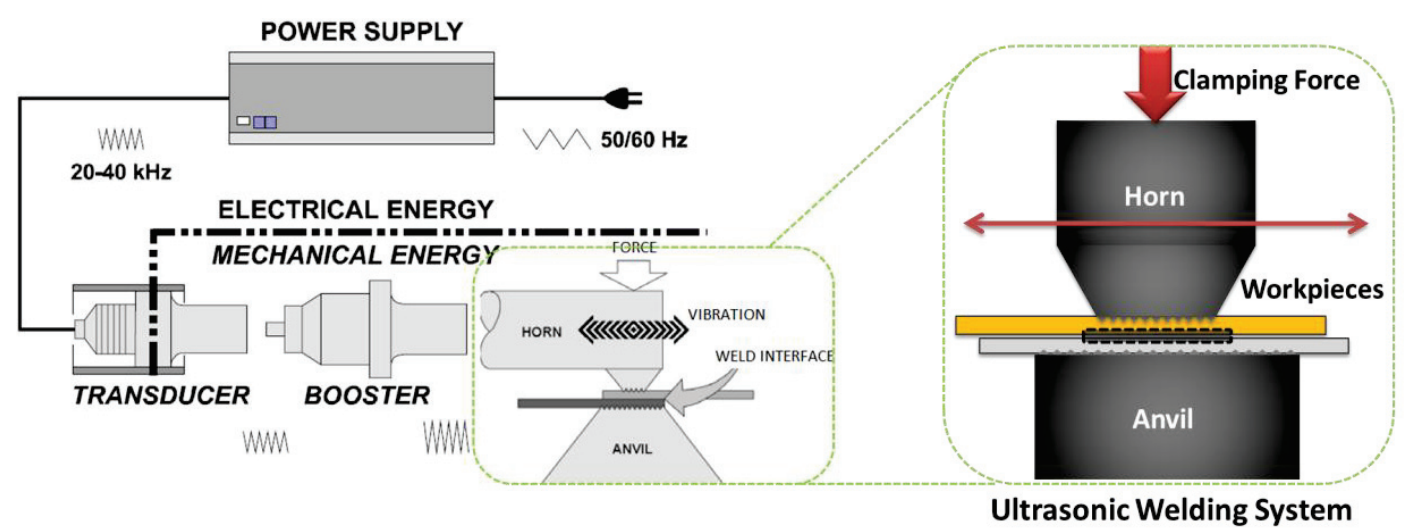

Figure 1. Ultrasonic welding system $[1,2]$

Ultrasonic metal welding is used in joining lithium-ion batteries due to its advantages in joining dissimilar and conductive materials, as discussed by Kim et al. [3]. Ultrasonic metal welding is a solid-state bonding process which uses high frequency ultrasonic energy to generate oscillating shears between two metal sheets clamped under pressure [1]. After removing the surface films and oxides from the surface, the solid-state bond is formed through the plastic deformation of the contacting surfaces under high pressure [4]. As illustrated in Figure 1, during welding, the transducer transforms electrical energy into high frequency mechanical vibration; this mechanical vibration is transferred to a welding tip through an acoustically tuned horn. This 
high frequency vibration, applied under force, disperses surface films and oxides, creating a metallurgical bond (Ultraweld $®$ by Branson Ultrasonics Corporation [2]).

The performance of an entire battery pack may not be as desired if any battery joint has a low quality connection. In order to ensure joint quality and not to pass any problematic weld to downstream processes, in a typical battery assembly plant, the quality of every single joint is inspected after the welding process through off-line manual inspection. This leads to delayed detection of low quality welds and a high manual inspection rate. The off-line quality inspection is a complex procedure that requires human operations and considerable time and labor in (a) visual inspection to ensure the welding spot is at the correct location, and (b) non-destructive mechanical test to ensure the bonding has sufficient strength. The cost of inspection becomes enormous when $100 \%$ manual inspection is performed on all welds. Therefore, the battery manufacturing processes used to join battery cells and modules need to be equipped with online real-time quality monitoring and evaluation systems to ensure the quality of joining. This motivates our research to develop an online monitoring system for ultrasonic battery tab welding that can help reduce unnecessary manual inspection rate and ensure the quality of every weld. The monitoring system predicts the quality of each weld based on real-time sensor signals collected from the ultrasonic welding process.

Weld quality has been classified into cold welds, good welds, and over welds by Kim et al. [3] through post-weld studies using the T-peel method. Both cold welds and over welds are considered problematic. Good welds have high peel strengths while problematic welds have low or medium peel strengths. We have observed from lab experiments and plant reports that a normal welding process, although with the presence of inherent variations, usually produces good welds. When the welding process is driven out-of-control due to assignable causes, e.g., 
metal surface contamination, improperly placed metal sheets, etc., bad welds are generated with a very high probability. For example, when the sheet metal is contaminated with oil (or other substances), the welding power would not ramp up as a normal weld does, thus resulting in a poor quality connection; if the metal sheets are improperly placed between the horn and anvil, the weld spot may fall on the edge of the sheets, also resulting in a poor quality connection; if one of the layers is bend when placing the sheets between the horn and anvil, the original input pressure may not be sufficient to make a strong connection on such an abnormal thickness. Therefore, it is important to detect process changes so that whenever the monitoring system detects an out-of-control sample, it would send a signal alarm to the downstream manual inspection, and the quality of that sample would then be verified by inspection.

Two types of errors may be committed by the monitoring system: false alarm, also known as the Type I error in hypothesis testing, and misdetection, also known as the Type II error. Specifically in this study, the Type I error occurs when the monitoring system announces a battery weld to be a suspect when it is actually in good quality; the Type II error occurs when the monitoring system fails to detect an out-of-control sample that turns out to be problematic. Thus, Type I error from the quality monitoring system results in unnecessary manual inspection efforts. On the other hand, passing a problematic weld will not only potentially impair the performance of the battery pack in electric vehicle, but also harm the performance and safety of the entire vehicle. Hence, Type II error results in passing problematic weld to downstream processes, which is a dangerous consequence that should be avoided. Therefore, the online quality monitoring system for ultrasonic welding of batteries needs to achieve a near-zero Type II error rate foremost while maintaining a relatively low Type I error rate in order to ensure weld quality and reduce manual inspection rate. 
Developing a monitoring system for ultrasonic welding of batteries that satisfies the above requirements on Type I and Type II errors is very challenging. The smallest Type I error and the smallest Type II error cannot be achieved at the same time due to the trade-off between risks of getting these two types of errors. When a broader acceptance region is defined, it would reduce false alarms but increase misdetections; on the other hand, a narrower acceptance region reduces the risks of misdetection, but this increases the number of false alarms. The conventional control chart techniques widely used in many process monitoring systems are designed to target a required Type I error rate. In operations where part quality is critical, a near-zero Type II error rate becomes the major goal for the monitoring system. It also needs a low Type I error to reduce unnecessary manual inspections, but even a relatively high Type I error rate (e.g. 50\%) represents a substantial reduction in manual inspection. Furthermore, the high frequency and short duration of ultrasonic welding process requires the real-time monitoring algorithm to be computationally efficient and its results to have good interpretability.

The objective of this article is to develop a monitoring algorithm that targets a near-zero Type II error rate foremost while maintaining a relatively low Type I error rate for the online quality monitoring system for ultrasonic welding of batteries. Specifically, the development of such a monitoring algorithm needs to effectively utilize sensor signals and integrate univariate and multivariate statistical process control methods. The developed monitoring algorithm will be used to help ensure part quality and reduce manual inspection costs in battery joining process and other mission-critical manufacturing processes as well. The remainder of this section briefly reviews existing methods on statistical process control. Section 2 describes the data collection procedure and the data and features used in this study. Detailed methodology on the proposed monitoring algorithm is presented in Section 3. Section 4 further demonstrates how the proposed 
monitoring algorithm works with a case study followed by a discussion in Section 5. Our conclusion is drawn in Section 6.

\subsection{Literature review of the related work}

In this study, the primary target of the monitoring system is to accurately identify the out-ofcontrol states of the welding process, rather than to distinguish problematic welds that are generated from an in-control process with common-cause variation. The importance of identifying the out-of-control states lies in that out-of-control processes are highly likely to produce problematic welds. Most of the problematic welds encountered in our study are attributed to some assignable root causes, or equivalently, special-cause variation, as previously explained. As such, a statistical process control approach is adopted in this research with the goal of reducing the Type I error while ensuring a near-zero Type II error. In this subsection, we will review the state of the art in the area of statistical process control.

In advanced manufacturing processes when near-zero Type II error rate is required, automated measurement technology is used, and every unit manufactured is analyzed. The univariate Shewhart control chart for individual measurements [5] is useful in such situations for separating assignable causes from chance causes. The individuals control chart detects out-ofcontrol samples by setting $k$-sigma control limits with

$$
\hat{\sigma}=\frac{\overline{M R}}{d_{2}},
$$

where $\overline{M R}$ is the average of the moving ranges of two observations, $d_{2}$ is a constant representing the expected value of the relative range, and $d_{2}=1.128$ when a moving range of 2 observations is used. This method is based on the assumption that the observations follow a normal 
distribution. In many practical scenarios, however, the normality assumption cannot be met. Borror et al. [6] found that the in-control average run length (ARL) is dramatically affected by non-normal data. One approach to dealing with the problem of non-normality is to determine the control limits for the individuals control chart based on the percentiles of the correct underlying distribution [7]. These percentiles could be obtained from a histogram when a large sample of observations is available.

Another limitation of the Shewhart individuals control chart is the restriction on the univariate perspective; that is, it is assumed that there is only one process output variable or quality characteristic of interest, or independence can be assumed among multiple output variables. In practice, however, most process monitoring and control scenarios involve several related variables, especially with the advances in sensing and data capture technology in recent decades. Although applying univariate control charts to each individual variable is possible, this is in fact inefficient and can lead to erroneous conclusions with a huge risk of misdetections. The Hotelling $\mathrm{T}^{2}$ control chart for monitoring the mean vector of the process is a multivariate version of the Shewhart control chart [8]. However, the Hotelling $\mathrm{T}^{2}$ control chart is based on the assumption that the observations follow multivariate normal distributions which may not hold in practice.

An exponentially weighted moving average (EWMA) control chart is robust to nonnormal distributions [6]. The multivariate exponentially weighted moving average (MEWMA) control chart is a multivariate version of EWMA control chart. The strength of MEWMA is in the capability of detecting small mean shifts $[5,9]$. However, MEWMA/EWMA charts are not advantageous in our problem due to the following reasons. First, MEWMA/EWMA charts are more effective than the Shewhart chart when the manufacturing process has a sustained small 
shift, which is not likely to exist in the ultrasonic welding process. A sustained shift usually results from tool wear, which is hardly a major concern in this study because the battery plant replaces tools based on a conservative strategy to minimize the effects of tool wear $[10,11]$. Additionally, when compared to Shewhart control charts, the results from EWMA/MEWMA charts are not straightforward to interpret, whereas from the perspective of plant implementation, it is desirable that monitoring results should be easily interpreted and the thresholds are straightforward to adjust.

Mahalanobis distance (M-distance) measures the similarity of an unknown data set to a known one based on correlations between them [12]. It differs from Euclidean distance in that it takes into account the correlations of the data set and is scale-invariant. As a multivariate control chart technique, M-distance can act as the control limit to detect multivariate out-of-control situations $[13,14]$. Moreover, M-distance method does not require the observations to follow normal distributions. Using a robust estimator of the covariance matrix in calculating the Mdistance would make the M-distance approach robust to non-normal distributed observations [15]. More studies on M-distance include Mitchell and Krzanowski [16], Barhen and Daudin [17], De Maesschalck et al. [14], Bedrick et al. [18], and Xiang et al. [19]. For manufacturing processes where the observations are not highly correlated or the correlation varies during the process, however, using M-distance alone has a huge risk of misdetection. Further considerations on this issue are discussed in Section 5.

In summary, multiple univariate Shewhart individuals control charts can be used together for process monitoring if the features are known to be independent, while the M-distance approach is a good choice if the features are known to be highly correlated. However, in many manufacturing processes, the correlation structure among the features is unknown and may be 
varying over time. Now that the strict quality requirement of near-zero Type II error rate becomes paramount, there is a lack of statistical process monitoring technique to fully address this goal, but the development of a new method by integrating multiple univariate Shewhart-type charts with M-distance holds promise.

\section{Data Collection Procedure and Data Description}

This section describes the data collection procedure and the data and features used in this study. The ultrasonic welding system is controlled by several input parameters including weld time, energy, maximum power, tool displacement before vibration, and tool displacement after vibration. The welding control system automatically obtains these features in order to check the status of the welding, i.e., whether or not the welder is operating properly as the input parameters specify. When the welding system fails to achieve a targeted input parameter, the system sends an alarm for the welding process. However, these features, although easily obtained, only provide limited information about the welding process, but fail to reflect many processes changes that are caused by assignable causes such as sheet surface contamination, fit-up weld, edge weld, etc., according to preliminary experiments. Therefore, the features provided by the welding system are not sensitive enough to separate problematic welds from high quality welds. Moreover, within-weld signals cannot be collected since these features do not show real-time information during the welding process. A preliminary analysis has shown that features from within-weld signals are important for process monitoring and quality prediction. For example, since the power signal rises as vibration starts and the surface films and oxides are removed from the surface, the slope of the rising of the power signal may be a good indicator of surface contamination, and some contamination may hinder the bonding of sheets and result in poor quality welds. 
In order to gather physical process information for weld quality monitoring, additional sensors including a watt (power) meter, a frequency sensor, and a linear variable differential transformer (LVDT) sensor are applied to collect both electrical and mechanical information during welding. Table 1 summarizes all the sensors, their signal type, and purpose. As shown in Figure 2, the watt meter and frequency sensors measure the transducer power and frequency at the weld control module. In the evaluation of an ultrasonic transducer for weld quality estimation, monitoring of the output ultrasonic power and frequency is important to understand and identify process and tool conditions during welding. The LVDT sensor is applied to measure displacement between the horn and anvil. Material deformation from LVDT signal profile is one of the most important process variables because plastic deformation as one of main bond mechanisms of ultrasonic welding is connected closely to material deformation. As described in the previous section, the ultrasonic metal welding system converts the periodic pulse of electrical power to mechanical oscillation through a piezo-ceramic module. Therefore, those three sensor signals can collect both electrical and mechanical information during welding. Preliminary welding experiments and post-weld studies have shown that certain features from these online signals can be correlated with joint quality. Although some of the correlated features are identified in Lee et al. [20], utilizing the signals systematically for weld quality monitoring remains a challenging task.
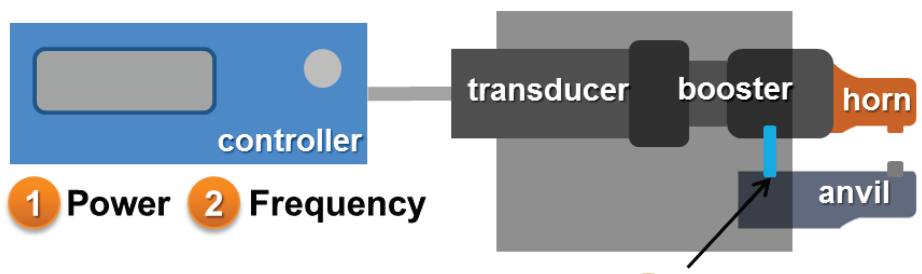

(3) LVDT

Figure 2. Sensors and their positions in the ultrasonic metal welding machine 
Table 1. Applied sensors, signal type, and purpose

\begin{tabular}{ccc}
\hline Sensor & Signal type & Purpose \\
\hline Watt (power) meter & $\begin{array}{c}\text { Ultrasonic power output } \\
\text { at piezo-ceramic module }\end{array}$ & Monitor controller power input signal \\
\hline Frequency sensor & $\begin{array}{c}\text { Frequency wave form } \\
\text { at piezo-ceramic module }\end{array}$ & $\begin{array}{c}\text { Measure frequency at the ultrasonic } \\
\text { transducer }\end{array}$ \\
\hline LVDT sensor & $\begin{array}{c}\text { Displacement } \\
\text { between horn and anvil }\end{array}$ & $\begin{array}{c}\text { Measure indentation and sheet thickness } \\
\text { variation during welding }\end{array}$ \\
\hline
\end{tabular}

Each signal can be divided into eight segments based on the different stages of the ultrasonic metal welding operation. Figure 3(a) shows each stage of the operation and the positions of the horn and anvil with respect to the battery tab. A welding operation starts when the battery tab is placed on the anvil (1) and the horn starts to move down (2). The horn then touches the sheet surface (3) and starts to vibrate (4). As the vibration continues, deformation layer grows and so does the weld area (5). After welding, vibration stops (6) and the horn lifts up (7) to its default position (8). Figure 3(b) and (c) show different segments of the LVDT signal and the power signal corresponding to the different stages of the ultrasonic metal welding operation respectively. The relationship between signal segments and operation stages is useful in interpreting the extracted features and relating them to the physics of the operation.

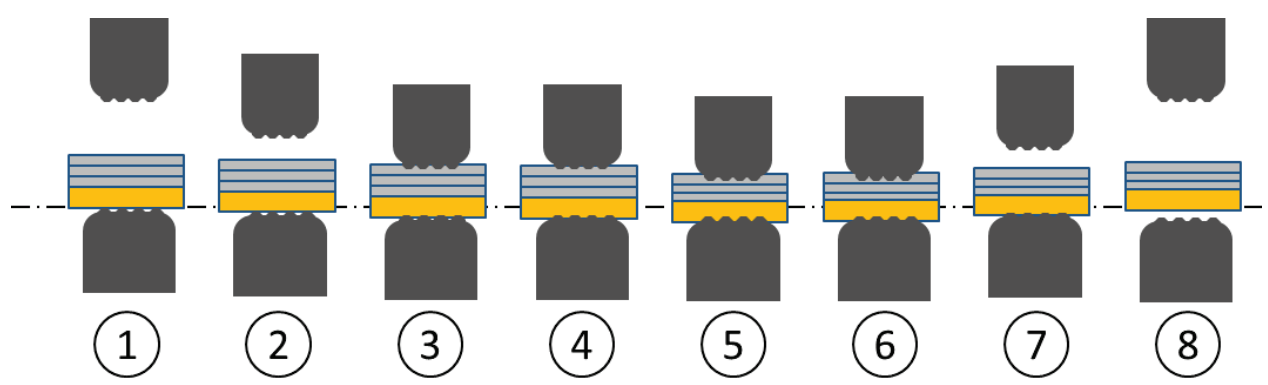

(a) Stages of ultrasonic metal welding operation 


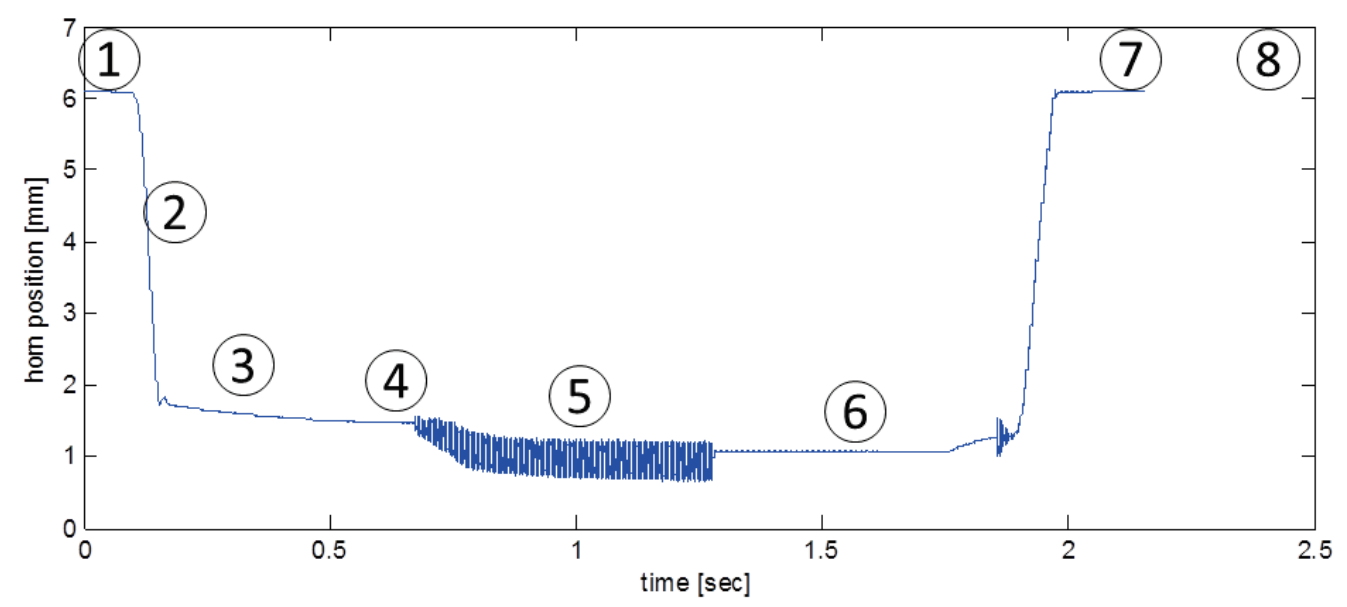

(b) Eight segments of LVDT signal

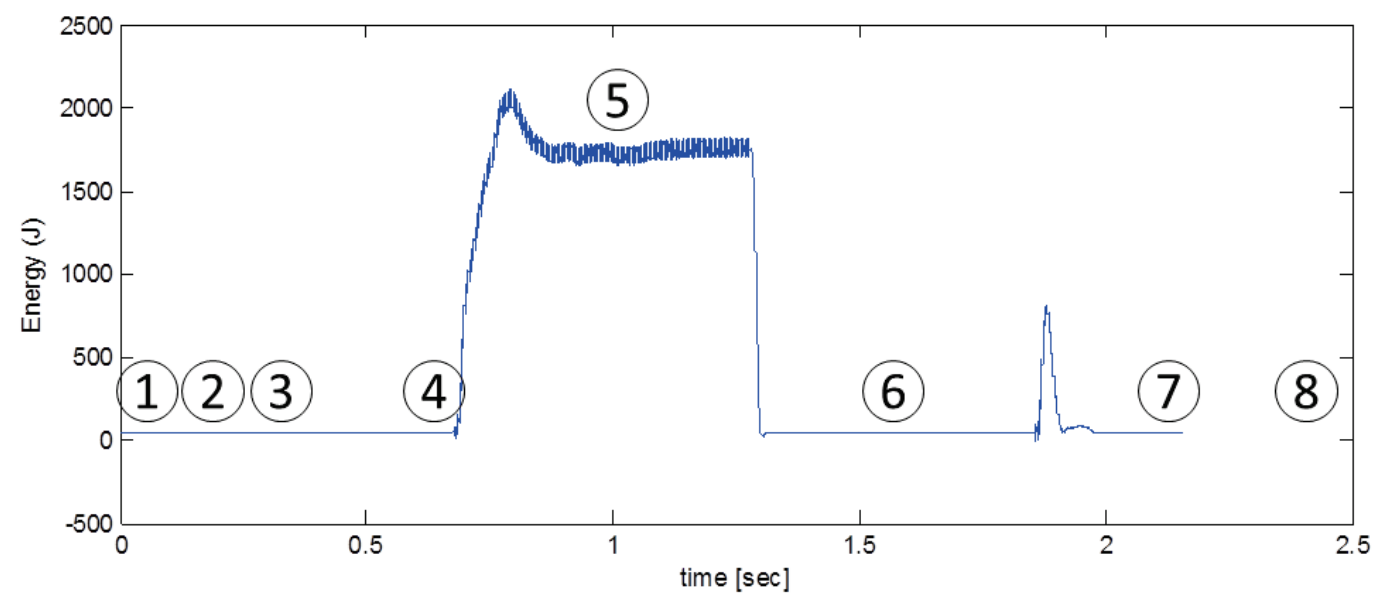

(c) Eight segments of power signal

Figure 3. Online signals and ultrasonic metal welding operation

Although the welding time is short, around $0.5 \mathrm{sec}$, measured signals have a lot of data due to the high sampling rate, $100 \mathrm{kHz}$. These data are too large to be completely utilized for process monitoring and some of the data from different signals can be redundant as they contain similar process information. Hence, the raw signal data should be transformed or reduced into a set of features by feature extraction which still contains sufficient accuracy to represent the 
welding process information with good separability between bad welds and good welds. The feature extraction consists of transforming, mapping, simplifying, and filtering. Ten features are then selected for process monitoring, as listed in Table 2. Details on the selection methods of these features can be found at Shao et al. [21].

Table 2. Features selected for process monitoring

\begin{tabular}{ccc}
$\begin{array}{c}\text { Feature } \\
\text { Index }\end{array}$ & Extracted From & Description \\
\hline F1 & LVDT signal & horn height before the main vibration \\
\hline F2 & LVDT signal & horn height after the main vibration \\
\hline F3 & power signal & maximum power value in the power signal \\
\hline F4 & welding control system & weld time during the main vibration \\
\hline F5 F9 & LVDT signal & \\
F10 & power signal & additional features \\
\hline
\end{tabular}

Training data was collected from an ultrasonic metal welding station over a 4-month period. Sensor signals were recorded and the features listed in Table 2 were extracted. Currently, most of welds produced are good and only a few cold welds are found occasionally. Thus, we focus on those cold welds as bad welds in our study. The total training data sample size is 23481 with 23437 good welds $(99.81 \%)$ and 44 bad welds $(0.19 \%)$. The small number of sample faults brings more challenges in establishing a threshold for the monitoring system. The proposed method was then tested on a 1-month period on the same station. The total test data sample size is 11507 with 11490 good welds (99.85\%) and 17 bad welds ( $0.15 \%)$. The Kolmogorov-Smirnov normality test on each feature gives a very small p-value (less than 0.01 ), which indicates that 
these features do not follow the normal distribution. Figure 4 shows the pre-processed values of feature F3 within a segment of the training period. Note these are pre-processed values showing the residuals of moving averages, instead of raw feature values. By taking the residuals of moving averages rather than the raw values in feature analysis, we can smooth out short-term fluctuations which are caused by material build-up, tool wear, etc. and highlight longer-term trends or cycles. A light grey dot in Figure 4 represents a good weld, while a dark circle represents a bad weld.

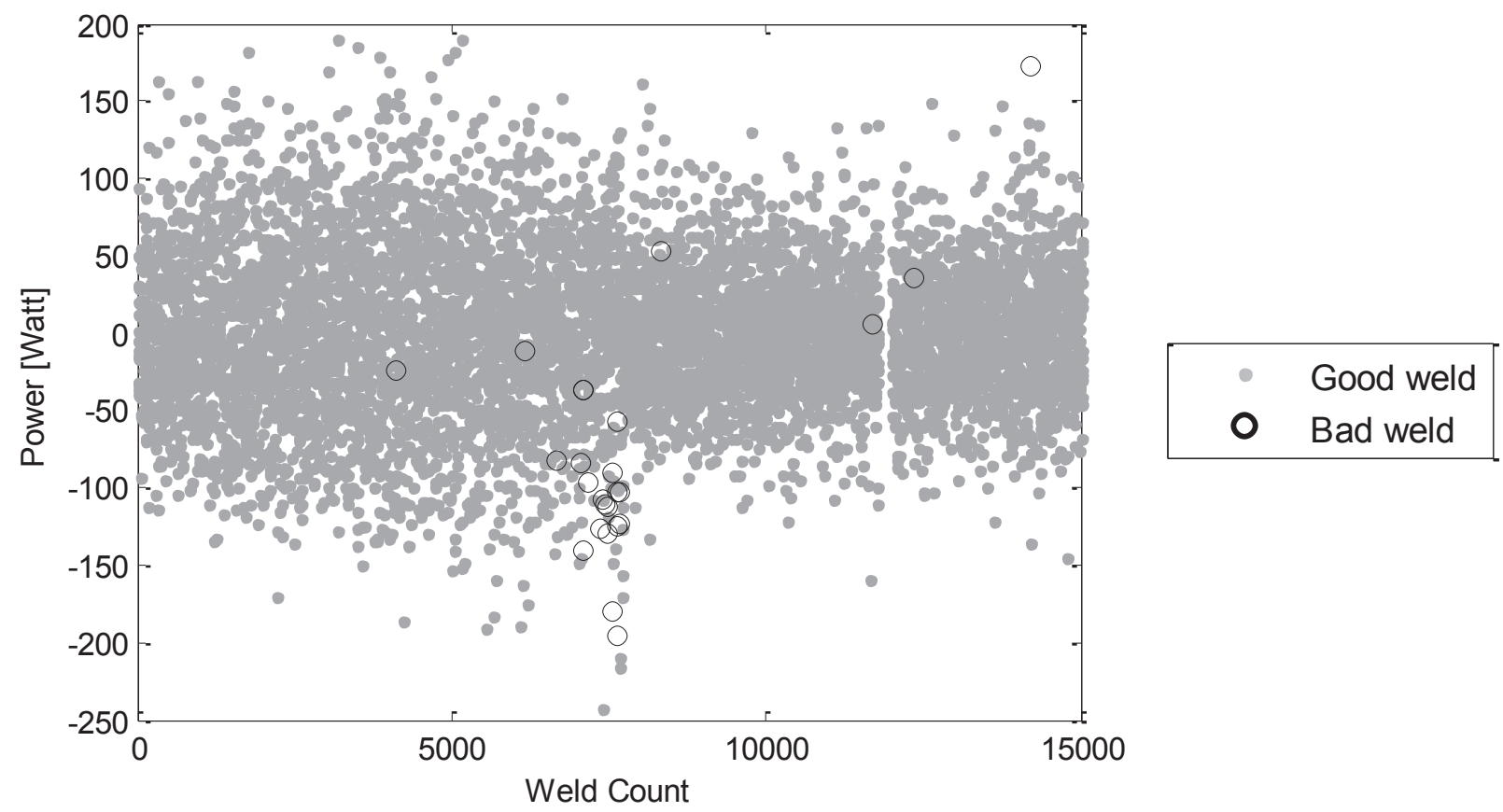

Figure 4. Distribution of feature F3 (maximum power value) in residuals of moving averages

The above datasets were collected along with a quality report from the $100 \%$ manual inspection in plant. The plant quality report provides information (good/bad) on the quality of each weld. Although the quality report serves as a baseline for developing monitoring system, the report may not be completely accurate due to possible operator errors. Hence, special care 
should be taken during monitoring algorithm development stage whenever the detection result disagrees with the quality report.

\section{Methodology}

\subsection{Overview of the proposed methodology}

The proposed method is depicted in the flowchart in Figure 5. The methodology consists of two main phases: (1) offline control limits training to achieve near-zero Type II error and (2) online quality monitoring using the tuned control limits. The "SPC-M algorithm" is developed by integrating univariate Shewhart-type control charts and the M-distance approach. In the training phase, the training dataset is used to construct the control limits for the SPC-M algorithm, which includes multiple univariate control limits for the Shewhart individuals control charts and control limits for the M-distance approach. The SPC-M control limits are tuned in order to achieve nearzero sample Type II error.

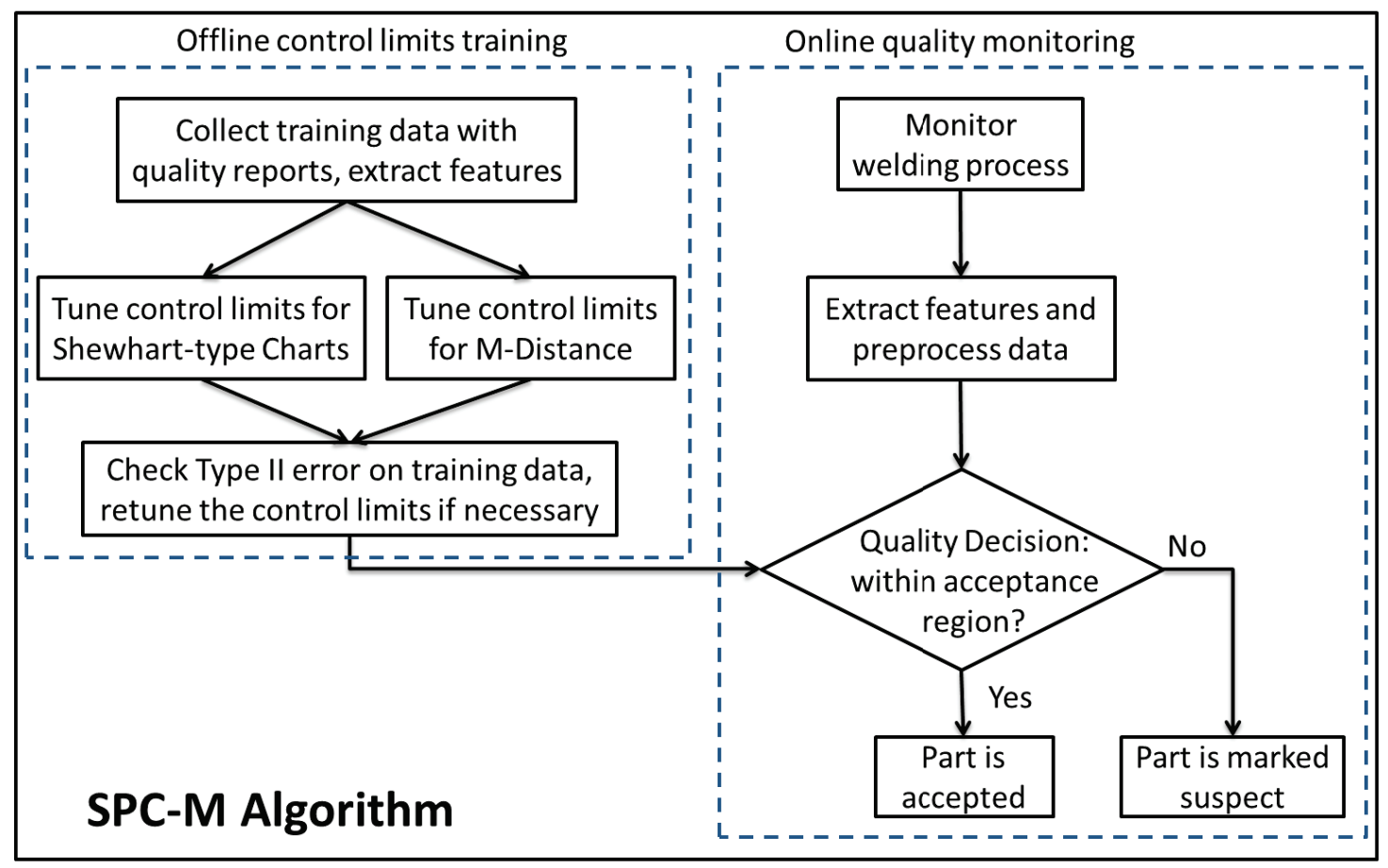


Figure 5. Overview of the proposed SPC-M methodology

In the second phase of online quality monitoring, welding process continues under the same operating condition as the previous period. For each test sample, features are extracted in the same way as discussed in Section 2. These features are then compared with the SPC-M control limits obtained from the training phase. If the test value falls into the acceptance region of SPC-M algorithm, we accept this weld as a good weld. Otherwise, the weld is marked as a suspect weld, and an alarm signal is generated. This weld will be sent to the manual inspection station for further quality check. The details of the SPC-M algorithm are explained in the following subsections.

\subsection{SPC-M algorithm}

The SPC-M algorithm is developed by integrating univariate Shewhart-type control charts and the M-distance approach to construct an integrated acceptance region. Let us look at a 2-feature case for example.

In Figure 6, the rectangles represent the acceptance regions given by a set of tight control limits and a set of slack control limits in the individuals control charts. For example, the tight acceptance region may be given by $1 \sigma$ control limits and the slack acceptance region may be given by $3 \sigma$ limits; the tight acceptance region may be given by 0.15 probability limits and the slack region may be given by 0.005 probability limits. The ellipse in Figure 6 represents the acceptance region given by a threshold from the multivariate M-distance approach. As illustrated in Figure 6, the acceptance region of the SPC-M algorithm consists of two regions, represented as $\mathrm{A}$ and $\mathrm{B}$. Region $\mathrm{A}$ is the tight acceptance region from the multiple univariate control limits in individuals control charts. If the features fall into this region, it indicates that all individual 
process variables are operated in a very tight range with very small variability, thus the process has little chance to produce defects. Region B is the region excluded from region A but governed by both the multivariate M-distance's threshold and a slack acceptance region from the multiple univariate control limits in individuals control charts. If the features fall into region $\mathrm{B}$, it indicates that their values exhibit variability but are acceptable in both univariate and multivariate aspects.

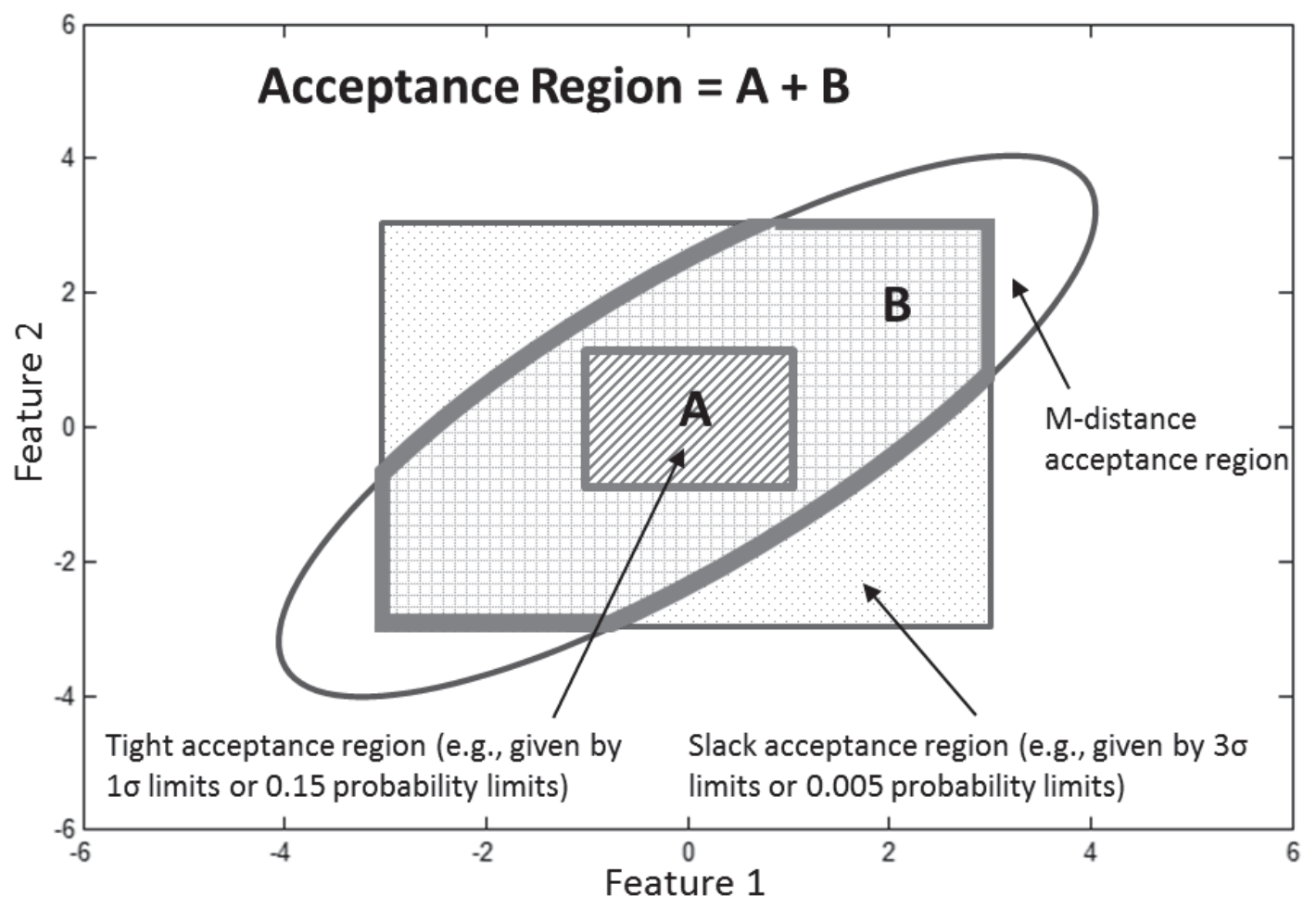

Figure 6. Acceptance region of SPC-M algorithm with $3 \sigma$ control limits

Considering data non-normality, we specify the control limits for individuals control charts by using probability limits instead of $k$-sigma limits in this article. For the M-distance approach, we adopt a robust estimator of covariance (Minimum Covariance Determinant (MCD) estimator, Rousseeuw (1984)) to make the method more robust to outliers and more reliable. The 
control limits are then tuned to achieve near-zero Type II error throughout the training period. Therefore, the integrated acceptance region, $\mathrm{A}+\mathrm{B}$, will be capable of monitoring multivariate observations with robustness to non-normal distributions and achieving near-zero Type II error rate. Details of the tuning of control limits are explained in the next subsection.

\subsection{Tuning control limits for the SPC-M algorithm}

Let $f$ denote the feature index, $f=1,2, \ldots, F$ and $F=10$ is the number of features in this study. Let $n_{g}$ denote the total number of good welds, $n_{b}$ denote the total number of bad welds in the training dataset; the total number of welds in the training dataset is

$$
n_{\text {training }}=n_{g}+n_{b}
$$

For the good welds, let $g_{i, f}$ denote the value of feature $f$ of weld $i, i=1,2, \ldots, n_{g}$. Then the values of feature $f$ of all good welds form a vector $G_{f}=\left[\begin{array}{llll}g_{1, f} & g_{2, f} & \cdots & g_{n_{g}, f}\end{array}\right]^{T}$. The feature values for all good welds can be represented as $G=\left[g_{i, f}\right]_{n_{g} \times F}$. Similarly, matrix $B=\left[b_{j, f}\right]_{n_{b} \times F}$ represents the feature values for all bad welds, where $b_{j, f}$ denotes the value of feature $f$ of weld $j$, $j=1,2, \ldots, n_{b}$. All training data can be represented as

$$
\mathrm{Y}_{\text {training }}=\left[\begin{array}{l}
G \\
B
\end{array}\right]=\left[y_{k, f}\right]_{n_{\text {training }} \times F}
$$

where $y_{k, f}$ denotes the value of feature $f$ of weld $k, k=1,2, \ldots, n_{\text {training }}$.

In setting up the acceptance region on univariate Shewhart-type control charts, each feature is considered individually. For $G_{f}$ of feature $f$, we construct univariate control limits 
$U C L_{1, f}, L C L_{1, f}, U C L_{2, f}$, and $L C L_{2, f}$ based on percentiles $p_{1 u}, p_{1 l}, p_{2 u}$, and $p_{2 l}$. As illustrated in Figure 7, $U C L_{1, f}$ and $L C L_{1, f}$ are the upper and lower control limits derived from percentile $\left(1-p_{1 u}\right)$ and $p_{1 l}$, respectively, and $U C L_{2, f}$ and $L C L_{2, f}$ are the upper and lower control limits derived from percentile $\left(1-p_{2 u}\right)$ and $p_{2 l}$, respectively. These two sets of control limits represent a tight acceptance region and a slack acceptance region in the univariate control charts. With the large number of training samples available, the percentile-based control limits can be obtained from the histogram of $G_{f}$. More specifically,

$$
\begin{gathered}
\left\{\begin{array}{l}
U C L_{1, f}=\left(1-p_{1 u}\right) \times 100^{\text {th }} \text { percentile of } G_{f} \\
L C L_{1, f}=\left(p_{1 l}\right) \times 100^{t h} \text { percentile of } G_{f}
\end{array},\right. \\
\left\{\begin{array}{l}
U C L_{2, f}=\left(1-p_{2 u}\right) \times 100^{t h} \text { percentile of } G_{f} \\
L C L_{2, f}=\left(p_{2 l}\right) \times 100^{t h} \text { percentile of } G_{f}
\end{array} .\right.
\end{gathered}
$$

For example, setting $p_{1 u}=p_{1 l}=0.1587$ and $p_{2 u}=p_{2 l}=0.00135$ correspond to the $1 \sigma$ and $3 \sigma$ control limits of Shewhart-type control limits under a normal distribution. For our training data, the univariate control limits can be determined by tuning $p_{1 u}, p_{1 l}, p_{2 u}$, and $p_{2 l}, p_{2 u} \leq$ $p_{1 u}, p_{2 l} \leq p_{1 l}$. Note that $p_{1 u}$ and $p_{1 l}, p_{2 u}$ and $p_{2 l}$ do not need to be set equal if the underlying distribution is not symmetric. 


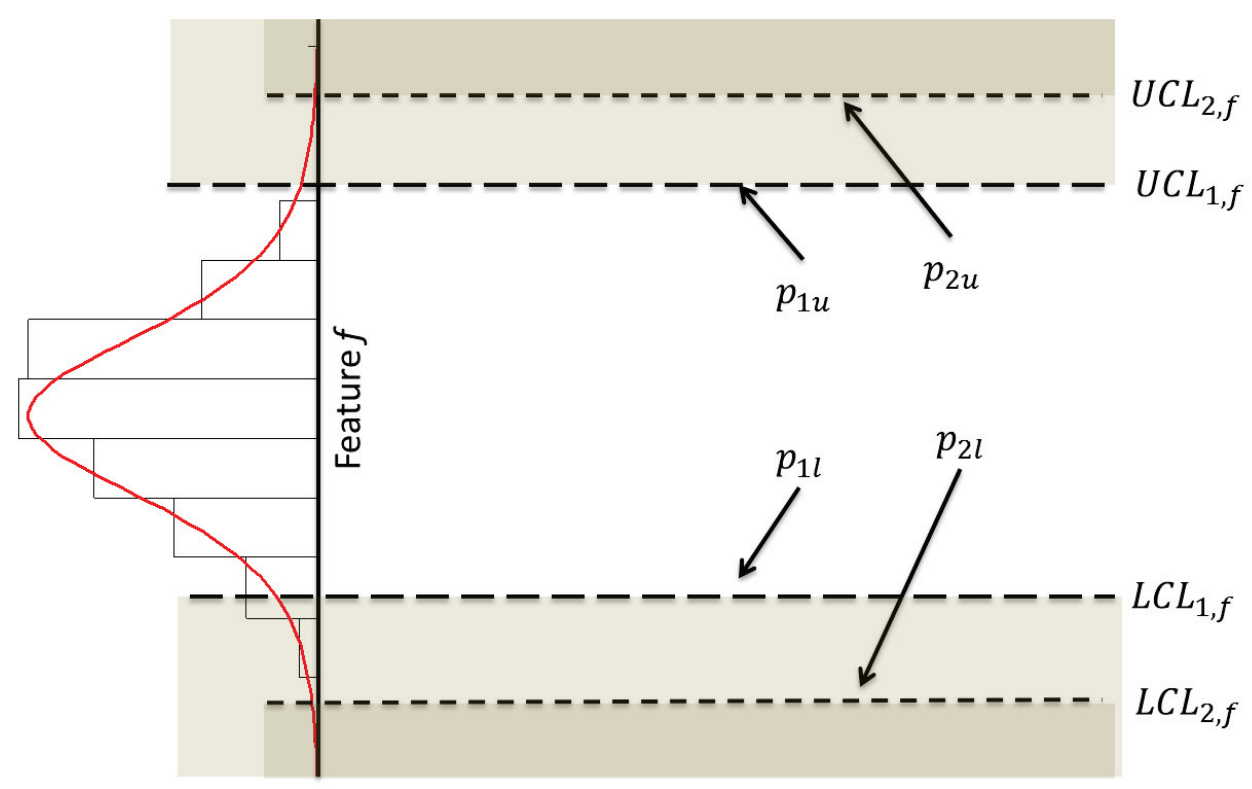

Figure 7. Univariate control limits used in SPC-M

Now we consider all features together and deal with multivariate data to set up the acceptance region on M-distance part. The multivariate feature value for a good sample $i$ in the training dataset is $x_{i}=\left[\begin{array}{llll}g_{i, 1} & g_{i, 2} & \cdots & g_{i, F}\end{array}\right]^{T}$. Let $\bar{G}_{f}$ denote the average value in $G_{f}$. Then the multivariate sample mean in the training dataset is $\bar{x}=\left[\begin{array}{llll}\bar{G}_{1} & \bar{G}_{2} & \ldots & \bar{G}_{F}\end{array}\right]^{T}$. A robust covariance $S$ is estimated using the Minimum Covariance Determinant, as explained by Rousseeuw (1984). The Mahalanobis distance of multivariate vector $x_{i}$ from the group of good samples with mean $\bar{x}$ and covariance matrix $S$ is given by

$$
d_{i}=\sqrt{\left(x_{i}-\bar{x}\right)^{T} \cdot S^{-1} \cdot\left(x_{i}-\bar{x}\right)} .
$$

The M-distance for all good welds in the training dataset is then given by $D_{g}=\left[\begin{array}{llll}d_{1} & d_{2} & \cdots & d_{n_{g}}\end{array}\right]^{T}$. The M-distance control limit $C L_{M}$ can be then constructed based on a percentile of $p_{M}$ with the histogram of $D_{g}$. More specifically, 


$$
C L_{M}=\left(1-p_{M}\right) \times 100^{t h} \text { percentile of } D_{g} .
$$

For our training data, we can tune $p_{M}$ to manipulate the control limit on M-distance, which is a multivariate measure of the features.

The parameters to be tuned in the training stage form a vector

$$
\boldsymbol{p}=\left(p_{1 u}, p_{1 l}, p_{2 u}, p_{2 l}, p_{M}\right)
$$

For the entire training dataset, $Y_{\text {training }}$ as defined in Eq. (3), we tune each element in $\boldsymbol{p}$ to achieve near-zero Type II error and also keep the Type I error as small as possible. The initial values of $\boldsymbol{p}$ may be set based on sample histogram. The SPC-M algorithm with control limits tuning are elaborated in Figure 8. The multiple univariate control limits $U C L_{1, f}, L C L_{1, f}, U C L_{2, f}$, and $L C L_{2, f}$, and the M-distance control limit $C L_{M}$ together establish the acceptance region for SPC-M. This integrated acceptance region is robust to weld quality and can be tuned to achieve zero Type II error. 


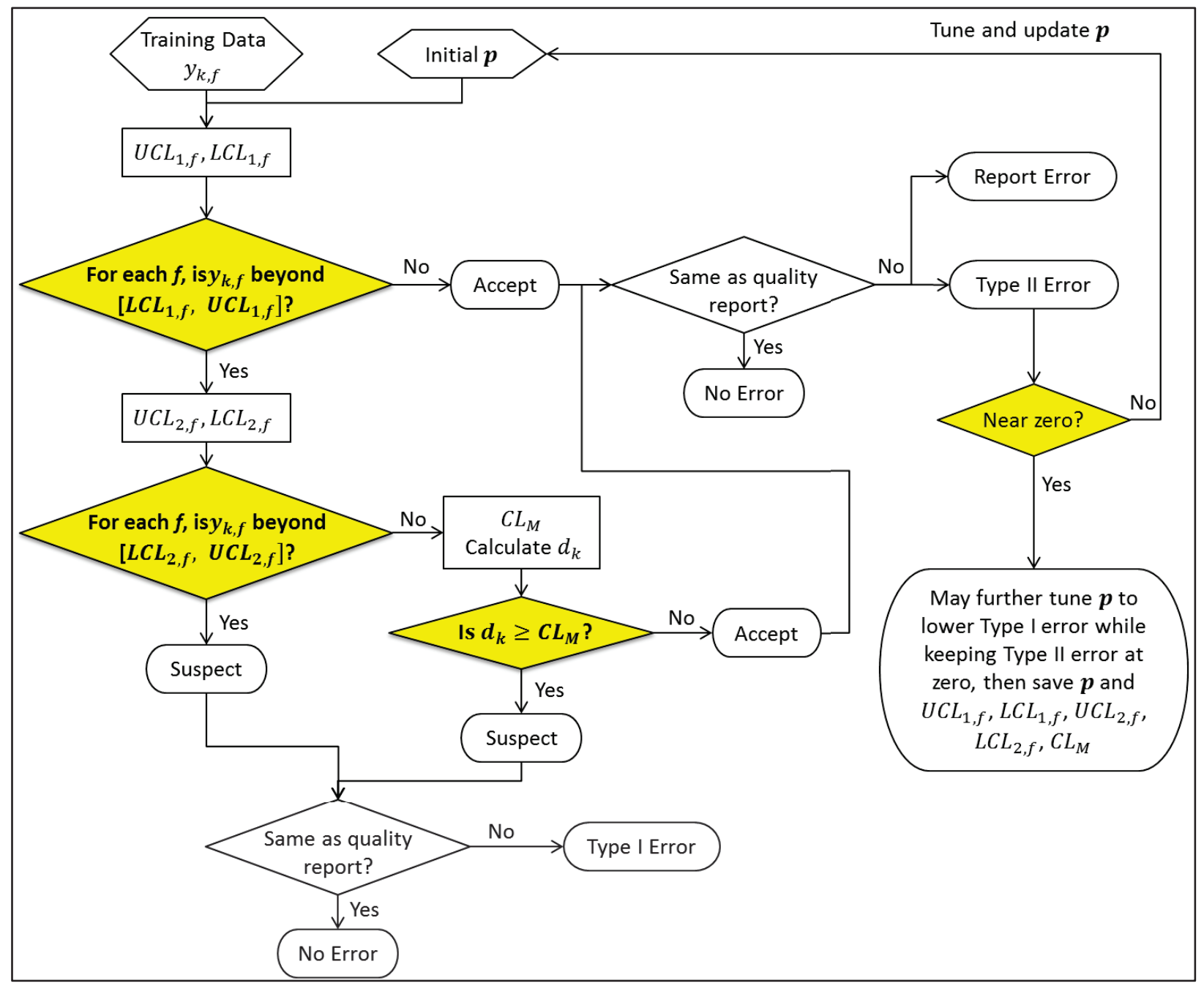

Figure 8. Flowchart of the SPC-M algorithm and control limits tuning

The multivariate feature value for a sample $k$ is $y_{k}=\left[\begin{array}{llll}y_{k, 1} & y_{k, 2} & \cdots & y_{k, F}\end{array}\right]^{T}$. For each feature $f$, we first compare $y_{k, f}$ with the tight control limits $\left[L C L_{1, f}, U C L_{1, f}\right]$ (similar to region A in Figure 6). If $y_{k, f}$ is within $\left[L C L_{1, f}, U C L_{1, f}\right]$ for all $f=1,2, \ldots, F$, we accept this weld $k$. If there is a $y_{k, f}$ beyond $\left[L C L_{1, f}, U C L_{1, f}\right]$, we then check it with the slack control limits $\left[L C L_{2, f}, U C L_{2, f}\right]$. If there is a $y_{k, f}$ beyond $\left[L C L_{2, f}, U C L_{2, f}\right]$, we then reject weld $k$ as a suspected problematic weld. Otherwise, we estimate the M-distance from weld $k$ to the group of good samples by 


$$
d_{k}=\sqrt{\left(y_{k}-\bar{x}\right)^{T} \cdot S^{-1} \cdot\left(y_{k}-\bar{x}\right)},
$$

and then check $d_{k}$ with the M-distance control limit $C L_{M}$. If $d_{k} \geq C L_{M}$, we reject weld $k$ and consider it as a suspected problematic weld; otherwise we accept it (similar to region B in Figure 6). After quality prediction using this set of parameters $\boldsymbol{p}$, we check the performance with quality reports to see whether Type II error is near-zero or not. Since the plant quality report may not be completely accurate, whenever a detection error is generated, detailed investigation will be performed through manual inspection and possible report errors will also be recorded. If not, we should tune $\boldsymbol{p}$ and update the acceptance region until near-zero Type II error is achieved. We may then try to further tune $\boldsymbol{p}$ to lower Type I error while keeping Type II error at zero.

\subsection{Testing the SPC-M algorithm}

Test data $\mathrm{Y}_{\text {test }}=\left[y_{k, f}\right]_{n_{\text {test }} \times F}$ is obtained as production continues, and $y_{k}=\left[\begin{array}{llll}y_{k, 1} & y_{k, 2} & \cdots & y_{k, F}\end{array}\right]^{T}$ is the multivariate feature value for weld $k$ in the test dataset. For each feature $f$, we compare $y_{k, f}$ with tight control limits $\left[L C L_{1, f}, U C L_{1, f}\right]$ and slack control limits $\left[L C L_{2, f}, U C L_{2, f}\right]$, which are obtained from previous analysis on training dataset. For each weld within $\left[L C L_{2, f}, U C L_{2, f}\right]$ but beyond $\left[L C L_{1, f}, U C L_{1, f}\right]$, we then check its M-distance with $C L_{M}$ obtained from training data analysis. The M-distance from weld $k$ to the group of good samples, $d_{k}$, is calculated according to Eq. (8), where $\bar{x}$ and $S$ are also obtained from the good samples in training data. Similar to the flowchart in Figure 8, a weld $k$ is rejected if there is a $y_{k, f}$ beyond $\left[L C L_{2, f}, U C L_{2, f}\right]$ or if $d_{k} \geq C L_{M}$

\section{Case Study}


In this section, we apply the SPC-M algorithm developed in the previous section to the dataset described in Section 2 and show the results in (1) designing control limits in the offline training stage and (2) evaluating monitoring performance.

\subsection{SPC-M algorithm design}

Following the developed SPC-M algorithm with our training dataset, we determined $\boldsymbol{p}$ to achieve zero Type II error. The percentile values are shown in Table 3.

Table 3. SPC-M algorithm parameters

\begin{tabular}{|l}
\hline M-distance threshold \\
\hline Univariate control charts limits
\end{tabular}

Tail probability: $p_{M}=0.08$

Tight limits with $p_{1 u}=p_{1 l}=0.15$

Slack limits with $p_{2 u}=p_{2 l}=0.005$

With this acceptance region, we can achieve an overall Type I error rate of $9.8 \%$ and a Type II error rate of $0 \%$ in the training dataset. Table 4 shows the training data performance in a confusion matrix. Among the 23437 good welds, 2290 welds are detected as problematic from our SPC-M algorithm, resulting in an overall Type I error rate of 9.8\%. Our algorithm detects all the 44 bad welds with zero Type II error.

Table 4. Confusion matrix for training data

\begin{tabular}{llllll} 
& & \multicolumn{2}{l}{ Predicted quality } & \multirow{2}{*}{ Overall detection error } \\
\cline { 2 - 5 } & & Good & Bad & Total & \\
\hline \multirow{3}{*}{ True quality } & Good & 21147 & 2290 & 23437 & \multirow{2}{*}{ Type I error rate $=\mathbf{9 . 8 \%}$} \\
\cline { 2 - 5 } & Bad & 0 & 44 & 44 & Type II error rate $=\mathbf{0 \%}$ \\
\cline { 2 - 5 } & Total & 21147 & 2334 & 23481 & \\
\hline
\end{tabular}


We would like to recommend a few guidelines to help with parameter tuning. (1) For univariate control limits, the tail probability for the tight limits should be between 0.05 and 0.4 $\left(0.05 \leq p_{1 u}, p_{1 l} \leq 0.4\right)$, whereas the tail probability for the slack limits should be between 0 and $0.05\left(0 \leq p_{2 u}, p_{2 l} \leq 0.05\right)$. A good starting point for $\left\{p_{1 u}, p_{1 l}, p_{2 u}, p_{2 l}\right\}$ would be $p_{1 u}=p_{1 l}=$ 0.1587 and $p_{2 u}=p_{2 l}=0.0062$, which are equivalent to the $1 \sigma$ and $3 \sigma$ limits in traditional Shewhart-type control charts. (2) Among all parameter candidates that achieve zero Type II error in the method training stage, we select the set of parameters that reduces the training Type I error rate to a certain extent. Minimizing the training Type I error is not a rigid objective in parameter tuning due to three reasons: first, the computational complexity brought by the optimization problem is high; second, it is desirable to avoid over-tuned parameters; and third, even a relatively high Type I error rate (e.g. 50\%) represents a substantial reduction in manual inspection.

\subsection{Monitoring performance evaluation}

As mentioned in Section 2, the sample size of test data is 11507 with 11490 good welds (99.85\%) and 17 bad welds $(0.15 \%)$. With the SPC-M algorithm parameters in Table 3, a zero Type II error rate was achieved through testing period, as shown in the confusion matrix in Table 5. Among the 11490 good welds, 2109 welds are detected as problematic from our algorithm, resulting in an overall Type I error rate of $18.4 \%$.

Table 5. Confusion matrix for test data

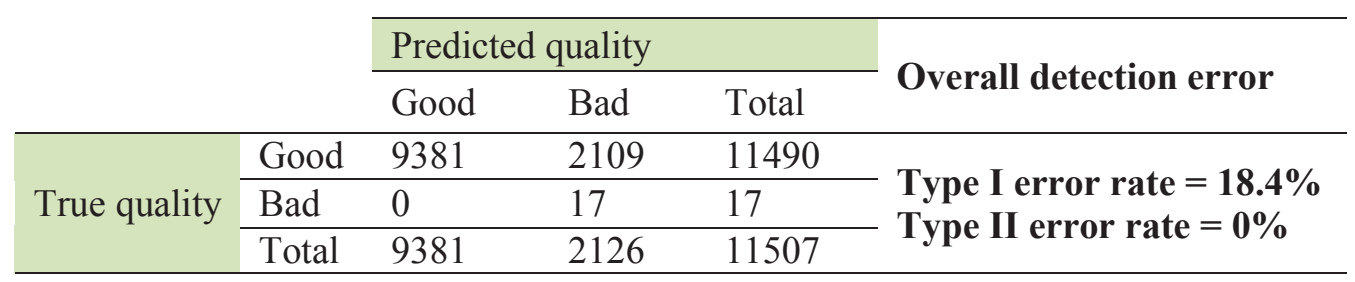




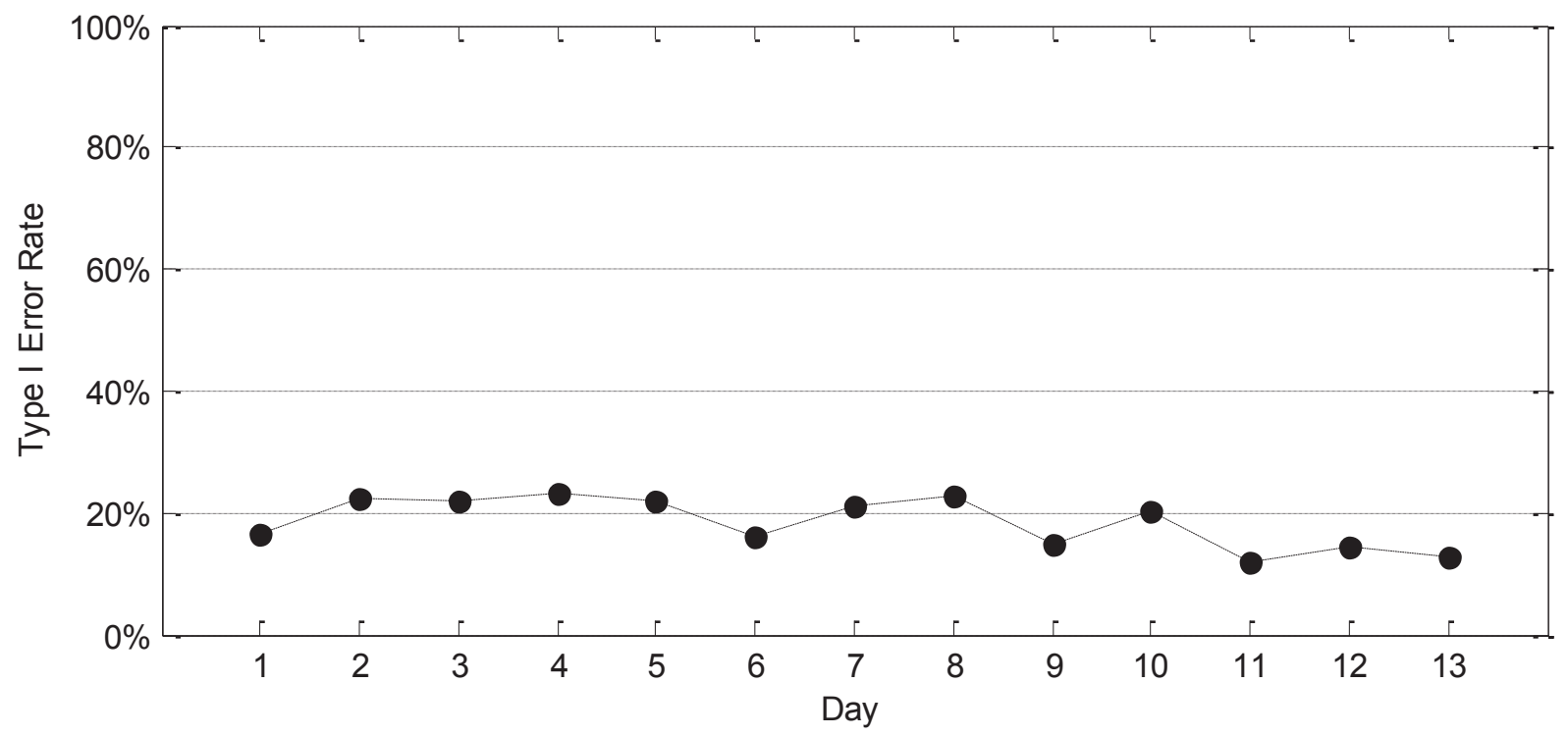

Figure 9. Daily Type I error rate through testing period while maintaining zero Type II error

Figure 9 shows the daily Type I error rate through the testing period. The maximum daily Type I error rate is less than $30 \%$ and the minimum daily Type I error rate is around $15 \%$. We know that the ultrasonic process is influenced by various factors such as tool wear, surface condition of the workpiece, and mechanical constraint of the workpiece. These factors cause the variability in Type I error rates. Even with this variability, the manual inspection rate is reduced more than $70 \%$ without changing tuning parameters of the SPC-M algorithm. More importantly, the SPC-M algorithm did not accept any bad welds in the testing period. Although the number of bad welds varied every day, a zero Type II error rate was achieved (all 17 bad welds were detected). This indicates that the proposed method performs very well in both the training and the testing periods.

Table 6. Performance comparison: SPC and M-distance 


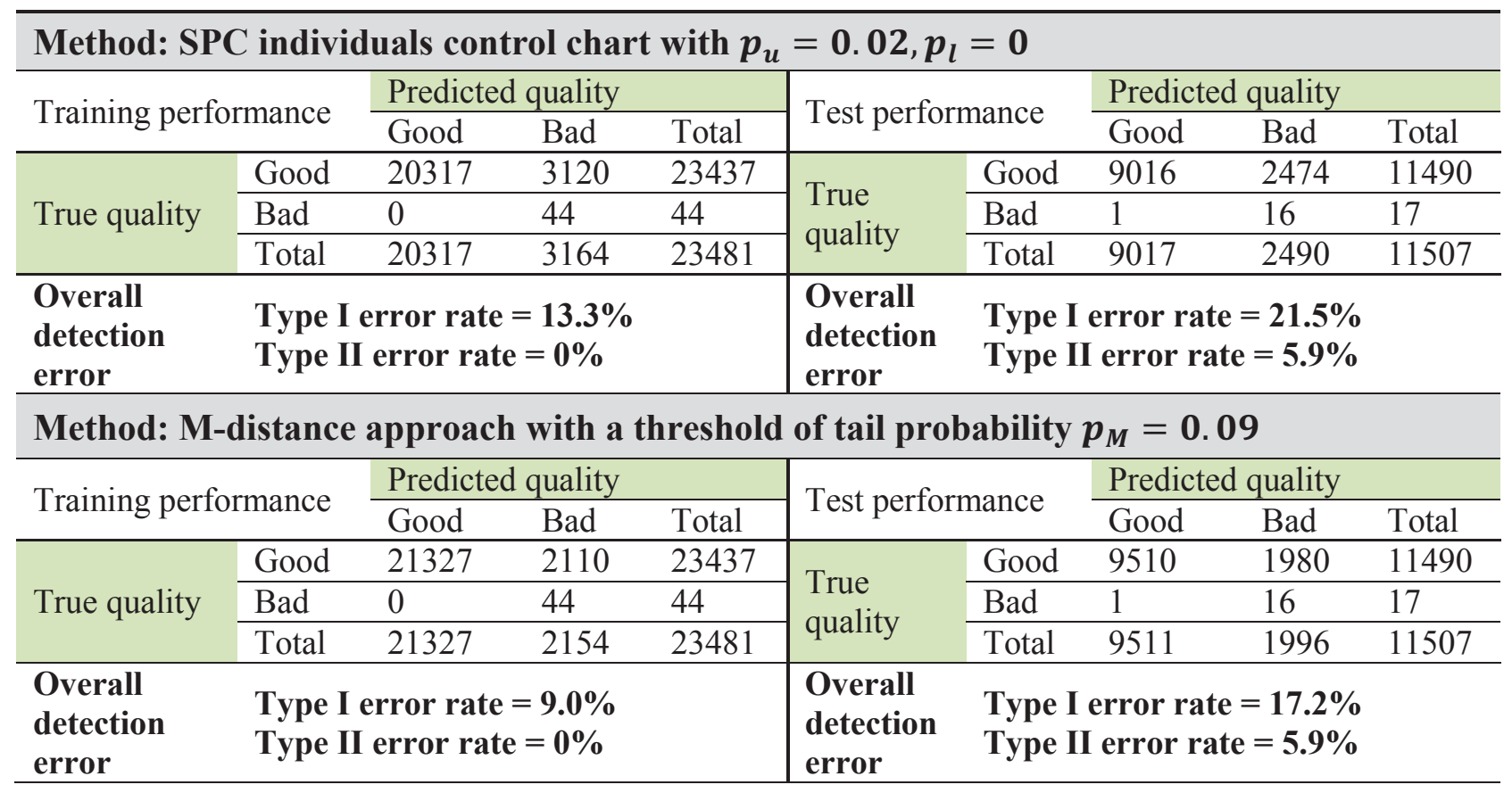

We further conducted a comparative study using either individuals control chart or Mdistance approach alone. The control limits were tuned so that zero Type II error can be achieved in training period. Among all parameter candidates that meet this criterion, we selected the parameter(s) that can minimize the training Type I error rate. Table 6 shows the training performance and test performance in the comparative study. Using the SPC individuals control charts alone, the Type I error rates in both training and testing periods are larger than those from SPC-M algorithm, whereas the Type I error rate from M-distance approach alone is slightly smaller than that from SPC-M. However, both these competitor methods have incurred huge costs when one bad weld in the testing period is misdetected. Comparing Table 6 with Table 4 and Table 5 demonstrates the superiority of using SPC-M algorithm in our case over SPC individuals control charts or M-distance approach. We have provided sample code and datasets of SPC-M algorithm at https://sites.google.com/site/weihonggraceguo/research/software, available for download. 


\section{Discussion}

The developed SPC-M algorithm has been demonstrated to work effectively on the ultrasonic welding of batteries. In this section, we further discuss the integrated algorithm so as to provide more guidelines to practitioners.

As mentioned in Section 3.3, the parameters to be tuned in the method training stage is a vector $\boldsymbol{p}=\left(p_{1 u}, p_{1 l}, p_{2 u}, p_{2 l}, p_{M}\right)$. Although the entire dataset has 10 features, it is assumed that the univariate control chart for each feature uses the same set of parameters $\left\{p_{1 u}, p_{1 l}, p_{2 u}, p_{2 l}\right\}$ to establish probability limits. It is feasible and applicable, however, to have different percentiles

for each feature, i.e., $\left\{p_{1 u}, p_{1 l}, p_{2 u}, p_{2 l}\right\}_{f=1}^{10}$, which can be tuned based on the feature's sensitivity. These percentiles could be obtained from a histogram if a large sample (at least 100 but preferably 200 observations) were available, or from a probability distribution fit to the data. For moderately small samples, probability plotting is an alternative to the histogram that can be used to determine the shape, center, and spread of the distribution. Intuitively, using different percentiles for each feature would give more flexibility in constructing the integrated acceptance region, and possibly more accurate detections. On the other hand, the disadvantages of introducing different sets of parameters for each feature are also obvious: the tuning of $40 \sim 50$ parameters simultaneously brings high computational complexity, probability limits in univariate control charts would be difficult to interpret, and that the tuned parameters may be overly casedependent and thus method generality is lost. Therefore, we would like to recommend $\boldsymbol{p}=$ $\left(p_{1 u}, p_{1 l}, p_{2 u}, p_{2 l}, p_{M}\right)$ as tuning parameters in the training stage, but we also acknowledge the feasibility of having more parameters in SPC-M. 
It is demonstrated in previous sections that the SPC-M algorithm is a data-driven technique targeting a near-zero sample Type II error rate and it is robust to non-normal distributed observations. The population error rate is estimated from the sample error rate based on training dataset and the control limits. In our case, the population Type I error rate is estimated as $\hat{\alpha}=1-\left(1-\hat{\alpha}_{I}\right)^{F} \cdot\left(1-\hat{\alpha}_{M}\right)=1-\left(1-p_{2}\right)^{10} \cdot\left(1-p_{M}\right)=16.8 \%$, where $\hat{\alpha}_{I}$ is the estimated sample Type I error rate from a Shewhart individuals chart and $\hat{\alpha}_{I}=p_{2 u}+p_{2 l} ; \hat{\alpha}_{M}$ is the estimated sample Type I error rate from the M-distance approach and $\hat{\alpha}_{M}=p_{M}$. This gives a general idea of the false alarm rate when a near-zero sample Type II error rate is achieved. Furthermore, since SPC-M control limits are determined to ensure the detection of all available defects in the training phase, the proposed algorithm is able to detect changes in variable correlation as well.

\section{Conclusion}

In this study, we address a critical issue in weld quality monitoring with near-zero Type II error rate and low manual inspection rate for ultrasonic metal welding process in a battery assembly plant. The conventional control chart techniques cannot achieve the near-zero Type II error rate that is desired in monitoring the battery joining process. To deal with this problem, we developed an SPC-M monitoring algorithm by integrating univariate statistical process control method and the Mahalanobis distance approach. The SPC-M algorithm has an integrated acceptance region which is the shared region of multiple univariate control limits and the Mahalanobis distance limits. In this way, the monitoring algorithm can be used to monitor multivariate processes in order to achieve near-zero Type II error rate. The control limits for SPC-M algorithm was tuned based on training data. Then the algorithm was validated on test data from battery manufacturing. The results showed that the SPC-M algorithm achieved a 21.6\% Type I error rate and 0\% Type II 
error rate overall. Comparative studies also demonstrated the superiority of using SPC-M algorithm in our case over SPC individuals control chart or M-distance approach. With 0\% Type II error rate, the SPC-M algorithm did not pass any suspected bad welds to downstream processes. The monitoring algorithm also proves to be robust against process variations such as tool wear, surface condition of the workpiece, and mechanical constraint of the workpiece, as evidenced by the $0 \%$ overall Type II error rate over the testing period.

In our study, the size of the training dataset is sufficiently large thanks to the rapid production rate in plant. Generally, 200 good samples are needed to find the control limits based on percentiles obtained from a histogram. However, a certain number of poor quality samples are also needed to help tuning the control limits to achieve near-zero Type II error rate. It is straightforward that the more poor quality samples there are in the offline training stage, the better the control limits will be tuned for the online monitoring stage. Therefore, developing an adaptive training scheme for SPC-M has the potential to shorten the algorithm training period and reduce data collection cost, and thus is an interesting topic for future research. Furthermore, associating detection errors with cost analysis would be a valuable development for online monitoring that is worth future research efforts.

Utilizing this SPC-M algorithm, the near-zero misdetection monitoring system can be applied to the monitoring of many mission-critical processes. The development of the SPC-M algorithm should assist manufacturing and quality engineers and in their decisions to specify good welds and problematic welds in a more cost-effective manner. 


\section{Acknowledgement}

This material is based upon work supported by the Department of Energy under Award Number DE-EE0002217. The authors would like to thank Drs. Mike Wincek, Kamran Paynabar, Juil Yum, Elijah Kannatey-Asibu, and Jeff Abell for their help with process understanding and data collection.

\section{Disclaimer}

This report was prepared as an account of work sponsored by an agency of the United States Government. Neither the United States Government nor any agency thereof, nor any of their employees, makes any warranty, express or implied, or assumes any legal liability or responsibility for the accuracy, completeness, or usefulness of any information, apparatus, product, or process disclosed, or represents that its use would not infringe privately owned rights. Reference herein to any specific commercial product, process, or service by trade name, trademark, manufacturer, or otherwise does not necessarily constitute or imply its endorsement, recommendation, or favoring by the United States Government or any agency thereof. The views and opinions of authors expressed herein do not necessarily state or reflect those of the United States Government or any agency thereof. 


\section{References}

[1] S.S. Lee, T.H. Kim, S.J. Hu, W.W. Cai, J.A. Abell, Joining Technologies for Automotive Lithium-ion Battery Manufacturing - A Review, in: ASME 2010 International Manufacturing Science and Engineering Conference, 2010.

[2] BRANSON, BRANSON Ultraweld® L20. BRANSON Ultrasonics Corporation, in: http://www.bransonultrasonics.com, 2007.

[3] T.H. Kim, J. Yum, S.J. Hu, J.P. Spicer, J.A. Abell, Process robustness of single lap ultrasonic welding of thin, dissimilar materials, CIRP Annals - Manufacturing Technology, 60 (2011) 1720.

[4] S. Kalpakjian, S.R. Schmid, Manufacturing processes for engineering materials, Pearson Education, Upper Saddle River, N.J., 2008.

[5] D.C. Montgomery, Introduction to statistical quality control, Wiley, Hoboken, NJ, 2013.

[6] C.M. Borror, D.C. Montgomery, G.C. Runger, Robustness of the EWMA control chart to non-normality, Journal of Quality Technology, 31 (1999) 309-316.

[7] T.R. Willemain, G.C. Runger, Designing control charts using an empirical reference distribution, Journal of Quality Technology, 28 (1996) 31.

[8] H. Hotelling, The Generalization of Student's Ratio, The Annals of Mathematical Statistics, 2 (1931) 360-378.

[9] Z.G. Stoumbos, J.H. Sullivan, Robustness to non-normality of the multivariate EWMA control chart, Journal of Quality Technology, 34 (2002) 260-276.

[10] C. Shao, W. Guo, T.H. Kim, J.J. Jin, S.J. Hu, J.P. Spicer, J.A. Abell, Characterization and monitoring of tool wear in ultrasonic metal welding, in: 9th International Workshop on Microfactories (IWMF2014), Honolulu, U.S.A., 2014.

[11] C. Shao, T.H. Kim, S.J. Hu, J. Jin, J. Abell, P. Spicer, Tool wear monitoring for ultrasonic metal welding of lithium-ion batteries, Journal of Manufacturing Science and Engineering, (2015).

[12] P.C. Mahalanobis, On the generalised distance in statistics, in: Proceedings National Institute of Science, India, 1936, pp. 49-55.

[13] S. Bersimis, S. Psarakis, J. Panaretos, Multivariate statistical process control charts: an overview, Quality and Reliability Engineering International, 23 (2007) 517-543.

[14] R. De Maesschalck, D. Jouan-Rimbaud, D.L. Massart, The mahalanobis distance, Chemometrics and Intelligent Laboratory Systems, 50 (2000) 1-18.

[15] P.J. Rousseeuw, Least Median of Squares Regression, Journal of the American Statistical Association, 79 (1984) 871-880.

[16] A.F.S. Mitchell, W.J. Krzanowski, The Mahalanobis Distance and Elliptic Distributions, Biometrika, 72 (1985) 464-467.

[17] A. Barhen, J.J. Daudin, Generalization of the Mahalanobis Distance in the Mixed Case, Journal of Multivariate Analysis, 53 (1995) 332-342.

[18] E.J. Bedrick, J. Lapidus, J.F. Powell, Estimating the Mahalanobis Distance from Mixed Continuous and Discrete Data, Biometrics, 56 (2000) 394-401.

[19] S. Xiang, F. Nie, C. Zhang, Learning a Mahalanobis distance metric for data clustering and classification, Pattern Recognition, 41 (2008) 3600-3612.

[20] S. Shawn Lee, C. Shao, T. Hyung Kim, S. Jack Hu, E. Kannatey-Asibu, W.W. Cai, J.

Patrick Spicer, J.A. Abell, Characterization of Ultrasonic Metal Welding by Correlating Online 
Sensor Signals With Weld Attributes, Journal of Manufacturing Science and Engineering, 136 (2014) 051019-051019.

[21] C. Shao, K. Paynabar, T.H. Kim, J. Jin, S.J. Hu, J.P. Spicer, H. Wang, J.A. Abell, Feature selection for manufacturing process monitoring using cross-validation, Journal of Manufacturing Systems, 32 (2013) 550-555. 


\section{Vitae}

Weihong Guo received her B.S. degree in Industrial Engineering from Tsinghua University, Beijing, China, in 2010 and an M.S. degree in Industrial and Operations Engineering from the University of Michigan, Ann Arbor, in 2012. She is currently a Ph.D. candidate in Industrial and Operations Engineering at the University of Michigan. Her research interests are in the areas of quality engineering and performance modeling for manufacturing and assembly systems using statistical process control techniques, machine learning methods, and the integration of SPC and automatic control. She is a member of INFORMS and IIE.

Chenhui Shao is a Ph.D. candidate in the Department of Mechanical Engineering at the University of Michigan, Ann Arbor. He received a B.E. degree in Automation from the University of Science and Technology of China in 2009, a M.S.E. degree in Industrial and Operations Engineering and a M.A. degree in Statistics from the University of Michigan, Ann Arbor in 2013. His research interests focus on quality engineering, manufacturing systems, and control theory. He is a member of ASME and IIE.

Tae Hyung Kim received the Ph.D. degree in precision mechanical engineering from Hanyang University, South Korea, in 2006. He is currently an Assistant Research Scientist of Mechanical Engineering at the University of Michigan. His research interests include lithium-ion battery manufacturing systems for electric vehicles, advanced joining technology for dissimilar materials, and intelligent monitoring and control systems in manufacturing processes.

S. Jack Hu received the B.S. degree from Tianjin University, Tianjin, China, in 1983, and the M.S. and Ph.D. degrees from the University of Michigan, Ann Arbor, in 1986 and 1990, respectively. He is currently a Professor of Mechanical Engineering and of Industrial and Operations Engineering. He conducts research and teaches courses in assembly, manufacturing systems, and statistical methods. He has published more than 200 papers in refereed journals of professional conferences. Dr. Hu is a Fellow of the American Society of Mechanical Engineers (ASME) and a Fellow of the International Academy for Production Engineering (CIRP).

Jionghua (Judy) Jin is a Professor in the Department of Industrial and Operations Engineering at the University of Michigan. She received her Ph.D. degree from the University of Michigan in 1999. Her research focuses on data fusion for improving system operational quality and efficiency. Her research emphasizes the integration of applied statistics, signal processing, reliability, system modeling and decision-making theory. She has received numerous awards including a NSF CAREER Award in 2002, a PECASE Award in 2004, and nine Best Paper Awards since 2005. She is a Fellow of IIE and ASME, and a senior member of ISI, a member of ASQ, IEEE, INFORMS, and SME.

J. Patrick Spicer is a staff researcher at General Motors Global Research and Development. Patrick holds a doctorate in manufacturing engineering, a masters in mechanical engineering and a bachelors in industrial and operations engineering, all from The University of Michigan. His research interests include battery manufacturing, manufacturing system design, cost modeling, and reconfigurable manufacturing systems.

Hui Wang is a researcher at General Motors Global Research and Development, Warren, Michigan. Hui holds a doctorate in Mechanical Engineering and Industrial \& Operations 
Engineering, a masters in Statistics from University of Michigan, and a bachelors in Mechanical Engineering from Tianjin University, China. His research interests include mathematical optimization, statistical modeling and their applications in battery manufacturing and manufacturing system design. 